\title{
MEAN DIMENSION AND AN EMBEDDING PROBLEM: AN EXAMPLE
}

\author{
ELON LINDENSTRAUSS, MASAKI TSUKAMOTO
}

\begin{abstract}
For any positive integer $D$, we construct a minimal dynamical system with mean dimension equal to $D / 2$ that cannot be embedded into $\left(\left([0,1]^{D}\right)^{\mathbb{Z}}\right.$, shift $)$.
\end{abstract}

\section{INTRODUCTION}

In this paper we study the problem of embedding a dynamical system $(X, T)$ (a compact metric space $X$ with a homeomorphism $T: X \rightarrow X)$ into $\left(\left([0,1]^{D}\right)^{\mathbb{Z}}, \sigma\right)$. Here $D$ is a positive integer, and $\sigma:\left([0,1]^{D}\right)^{\mathbb{Z}} \rightarrow\left([0,1]^{D}\right)^{\mathbb{Z}}$ is the shift transformation: $\sigma(x)_{n}=x_{n+1}$. "Embedding" means a topological embedding $f: X \rightarrow\left([0,1]^{D}\right)^{\mathbb{Z}}$ satisfying $f T=\sigma f$.

An obvious obstacle for the embedding of a dynamical system $(X, T)$ into $\left(\left([0,1]^{D}\right)^{\mathbb{Z}}, \sigma\right)$ is given by the set of periodic points of $X$ : if the set $\operatorname{Peri}_{n}(X, T)$ of periodic points of period $n$ cannot be topologically embedded into $[0,1]^{D n}$ for some $n$, then $(X, T)$ cannot be embedded into $\left(\left([0,1]^{D}\right)^{\mathbb{Z}}, \sigma\right)$ (an expanded discussion of this obstruction can be found in Gutman [4, Example 1.8].) When $X$ has finite topological dimension Jaworski [6] (see also Auslander [1, Chapter 13, Theorem 9]) proved that if $(X, T)$ has no periodic points then $(X, T)$ can be embedded into the system $\left([0,1]^{\mathbb{Z}}, \sigma\right)$. Our main concern in this paper is the case of $(X, T)$ minimal.

Mean dimension (denoted $\operatorname{mdim}(X, T)$ ) is a natural invariant of dynamical systems introduced by Gromov [3]. It is zero for finite dimensional systems, and is equal to $D$ for the dynamical system $\left(\left([0,1]^{D}\right)^{\mathbb{Z}}, \sigma\right)$. Weiss and the first named author observed in $[8]$ that mean dimension gives another, less obvious obstacle for embedding a dynamical system in another: namely, if $(X, T)$ can be embedded into the system $(Y, S)$ then $\operatorname{mim}(X, T) \leq$ $\operatorname{mdim}(Y, S)$. In particular, if $(X, T)$ can be embedded in $\left(\left([0,1]^{D}\right)^{\mathbb{Z}}, \sigma\right)$ then $\operatorname{mdim}(X, T) \leq$ $D$. A construction of a minimal dynamical system (which in particular has no periodic points) whose mean dimension is greater than 1 is given in [8, Proposition 3.3]; it follows that this system cannot be embedded into $\left([0,1]^{\mathbb{Z}}, \sigma\right)$ despite the fact that it has no periodic points.

Date: August 9, 2013.

2010 Mathematics Subject Classification. 37B99, 54F45.

Key words and phrases. Mean dimension.

Masaki Tsukamoto was supported by Grant-in-Aid for Young Scientists (B) (21740048). Elon Lindenstrauss acknowledges the support of the ISF and ERC. 
In [7] the first named author proved a partial converse to above necessary criterion for embeddedability of a dynamical system in $\left(\left([0,1]^{D}\right)^{\mathbb{Z}}, \sigma\right)$ :

Theorem 1.1. There exists a positive number $c \geq 1 / 36$ satisfying the following: If a dynamical system $(X, T)$ is an extension of an infinite minimal system and $\operatorname{mim}(X, T)<$ $c D$, then $(X, T)$ can be embedded into the system $\left(\left([0,1]^{D}\right)^{\mathbb{Z}}, \sigma\right)$.

This raises the interesting problems of determining the optimal value of the positive constant $c$ in the above statement.

Recall the following classical result in dimension theory ([5, p. 56, Theorem V 2]): If $X$ is a compact metric space with $\operatorname{dim} X \leq D$, then $X$ can be topologically embedded into $[0,1]^{2 D+1}$. This motivates the following conjecture:

Conjecture 1.2. Let $(X, T)$ be a dynamical system so that for every $n$ we have that $\frac{1}{n} \operatorname{dim}\left(\operatorname{Peri}_{n}(X, T)\right)<D / 2$ and $\operatorname{mdim}(X, T)<D / 2$. Then $(X, T)$ can be embedded into the system $\left(\left([0,1]^{D}\right)^{\mathbb{Z}}, \sigma\right)$.

The main result of this paper is the following.

Theorem 1.3. Let $D$ be a positive integer. There exists a minimal system $(X, T)$ with $\operatorname{mdim}(X, T)=D / 2$ but $(X, T)$ cannot be embedded into the system $\left(\left([0,1]^{D}\right)^{\mathbb{Z}}, \sigma\right)$.

This theorem shows that if Conjecture 1.2 is true then the condition $\operatorname{mim}(X, T)<D / 2$ is optimal.

Acknowledgments. We thank Gil Kalai for the reference to A. B. Skopenkov's paper [10] and Matoušek's book [9]. These references were the starting point for this work.

\section{SOME PRELIMINARIES}

2.1. Review of mean dimension. We review the basic definitions of mean dimension; cf. Gromov [3] and Lindenstrauss-Weiss [8] for more details. Let $(X, d)$ be a compact metric space. Let $Y$ be a topological space, and let $f: X \rightarrow Y$ be a continuous map. For a positive number $\varepsilon$, we call $f$ an $\varepsilon$-embedding if we have $\operatorname{Diam} f^{-1}(y) \leq \varepsilon$ for all $y \in Y$. We define $\operatorname{Widim}_{\varepsilon}(X, d)$ as the minimum integer $n \geq 0$ such that there exist an $n$ dimensional polyhedron (a topological space admitting a structure of simplicial complex) $P$ and an $\varepsilon$-embedding $f: X \rightarrow P$. The following example (cf. Gromov [3, p. 332]) will be used later; for a proof, see [8, Lemma 3.2]:

\section{Example 2.1.}

$$
\operatorname{Widim}_{\varepsilon}\left([0,1]^{N}, d_{\ell}\right)=N, \quad(0<\varepsilon<1),
$$

where $d_{\ell \infty}$ is the $\ell^{\infty}$-distance: $d_{\ell \infty}(x, y)=\max _{i}\left|x_{i}-y_{i}\right|$.

We also note the following lemma: 
Lemma 2.2. Let $(X, d)$ and $\left(Y, d^{\prime}\right)$ be compact metric spaces. Suppose that there is a continuous distance-increasing map from $X$ to $Y$. Then $_{\operatorname{Widim}}(X, d) \leq \operatorname{Widim}_{\varepsilon}\left(Y, d^{\prime}\right)$ for all $\varepsilon>0$. (A map $f: X \rightarrow Y$ is distance-increasing if $d(x, y) \leq d(f(x), f(y)$ ) for all $x, y \in X$.)

Proof. If $f: X \rightarrow Y$ is distance-increasing and $g: Y \rightarrow P$ is an $\varepsilon$-embedding, then $g \circ f: X \rightarrow P$ is also an $\varepsilon$-embedding.

Let $T: X \rightarrow X$ be a homeomorphism. For an integer $n \geq 0$, we define a distance $d_{n}$ on $X$ by $d_{n}(x, y)=\max _{0 \leq i<n} d\left(T^{i} x, T^{i} y\right)$. We define the mean dimension $\operatorname{mim}(X, T)$ by

$$
\operatorname{mdim}(X, T):=\lim _{\varepsilon \rightarrow 0}\left(\lim _{n \rightarrow \infty} \frac{\operatorname{Widim}_{\varepsilon}\left(X, d_{n}\right)}{n}\right) .
$$

The function $n \mapsto \operatorname{Widim}_{\varepsilon}\left(X, d_{n}\right)$ is subadditive. Hence the above limit exists. The mean dimension $\operatorname{mim}(X, T)$ is a topological invariant, i.e., it is independent of the choice of a distance $d$ compatible with the topology. The fundamental example is the following (for the proof, see [8, Proposition 3.3]):

Example 2.3. Let $D$ be a positive integer. Consider the $D$-dimensional unit cube $[0,1]^{D}$. Let $\left([0,1]^{D}\right)^{\mathbb{Z}}$ be the infinite product of the copies of $[0,1]^{D}$ indexed by $\mathbb{Z}$ with the product topology. Let $\sigma:\left([0,1]^{D}\right)^{\mathbb{Z}} \rightarrow\left([0,1]^{D}\right)^{\mathbb{Z}}$ be the shift transformation: $\sigma(x)_{n}=x_{n+1}$. Then

$$
\operatorname{mdim}\left(\left([0,1]^{D}\right)^{\mathbb{Z}}, \sigma\right)=D .
$$

In section 3, we use a "block-type" system. This type of construction was used in the context of mean dimension by Weiss and the first named author in [8, Proposition 3.5] and by Coornaert-Krieger [2]. Let $K$ be a compact metric space, and let $b$ be a positive integer. Let $B \subset K^{b}$ be a closed subset. We define a block-type system $X(B) \subset K^{\mathbb{Z}}$ by

$$
X(B):=\left\{x \in K^{\mathbb{Z}} \mid \exists k \in \mathbb{Z} \forall l \in \mathbb{Z}: x_{k+l b}^{k+l b+b-1} \in B\right\}
$$

where $x_{m}^{n}=\left(x_{m}, x_{m+1}, \ldots, x_{n}\right)$ for $m \leq n$. Let $\sigma: X(B) \rightarrow X(B)$ be the shift transformation.

\section{Lemma 2.4.}

$$
\operatorname{mdim}(X(B), \sigma) \leq \frac{\operatorname{dim} B}{b}
$$

Proof. Let $d$ be a distance on $K$. We define a distance $d^{\prime}$ on $K^{\mathbb{Z}}$, compatible with the product topology, by

$$
d^{\prime}(x, y):=\sum_{n \in \mathbb{Z}} 2^{-|n|} d\left(x_{n}, y_{n}\right) .
$$

For $\varepsilon>0$, we take a positive integer $L=L(\varepsilon)$ satisfying $\sum_{|n|>L} 2^{-|n|}<\varepsilon / \operatorname{Diam}(K)$. For a positive integer $n$, let $\pi_{n}: K^{\mathbb{Z}} \rightarrow K^{\{-L,-L+1, \ldots, n+L\}}$ be the natural projection. If two points $x, y \in K^{\mathbb{Z}}$ satisfy $\pi_{n}(x)=\pi_{n}(y)$, then $d_{n}^{\prime}(x, y)<\varepsilon$. 
We decompose $X(B): X(B)=X_{0} \cup X_{1} \cup \cdots \cup X_{b-1}$ where

$$
X_{k}=\left\{x \in K^{\mathbb{Z}} \mid \forall l \in \mathbb{Z}: x_{k+l b}^{k+l b+b-1} \in B\right\} .
$$

By definition,

$$
\operatorname{dim} \pi_{n}\left(X_{k}\right) \leq \frac{n \operatorname{dim} B}{b}+\text { const }
$$

where const is a positive constant independent of $n$. Recall the Sum Theorem in dimension theory [5, p. 30]: if a compact metric space $Y$ is a countable or finite union of closed sets $Y_{i}$ then $\operatorname{dim} Y=\max _{i} \operatorname{dim} Y_{i}$. Applying this theorem to the sets $X_{i}$ we obtain

$$
\operatorname{dim} \pi_{n}(X(B)) \leq \frac{n \operatorname{dim} B}{b}+\text { const. }
$$

Since $\pi_{n}: X(B) \rightarrow \pi_{n}(X(B))$ is an $\varepsilon$-embedding,

$$
\operatorname{Widim}_{\varepsilon}\left(X(B), d_{n}^{\prime}\right) \leq \operatorname{dim} \pi_{n}(X(B)) \leq \frac{n \operatorname{dim} B}{b}+\text { const. }
$$

Hence

$$
\operatorname{mdim}(X(B), \sigma)=\lim _{\varepsilon \rightarrow 0}\left(\lim _{n \rightarrow \infty} \frac{\operatorname{Widim}_{\varepsilon}\left(X(B), d_{n}^{\prime}\right)}{n}\right) \leq \frac{\operatorname{dim} B}{b}
$$

2.2. Topological preliminaries. First we fix some notations. For a topological space $X$ we define its cone $C(X)$ by $C(X):=[0,1] \times X / \sim$ where $(0, x) \sim(0, y)$ for all $x, y \in X$. The equivalence class of $(t, x)$ is denoted by $[t x]$. We set $\sigma_{n-1}$ to be the $(n-1)$-dimensional simplex

$$
\sigma_{n-1}:=\left\{\left(t_{1}, \ldots, t_{n}\right) \in \mathbb{R}^{n} \mid t_{1}, \ldots, t_{n} \geq 0, t_{1}+\cdots+t_{n}=1\right\} .
$$

For topological spaces $X_{1}, \ldots, X_{n}$ we define their join $X_{1} * \cdots * X_{n}$ by

$$
X_{1} * \cdots * X_{n}:=\sigma_{n-1} \times X_{1} \times \cdots \times X_{n} / \sim
$$

where $\left(t_{1}, \ldots, t_{n}, x_{1}, \ldots, x_{n}\right) \sim\left(s_{1}, \ldots, s_{n}, y_{1}, \ldots, y_{n}\right)$ iff

$$
t_{i}=s_{i}(\forall 1 \leq i \leq n) \quad \text { and } \quad x_{i}=y_{i}\left(\forall 1 \leq i \leq n \text { satisfying } t_{i} \neq 0\right) .
$$

The equivalence class of $\left(t_{1}, \ldots, t_{n}, x_{1}, \ldots, x_{n}\right)$ is denoted by $t_{1} x_{1} \oplus \cdots \oplus t_{n} x_{n}$. If $X_{1}, \ldots, X_{n}$ admit the structure of a simplicial complex, so does $X_{1} * \cdots * X_{n}$ in a canonical way.

Let $Y$ be the triod graph, i.e. the graph of the shape "Y". (Rigorous definition is as follows. Let $D_{3}$ be the 3-points discrete space, and set $Y:=C\left(D_{3}\right)$ : the cone of $D_{3}$.) Let $d$ be the graph distance on $Y$ (all three edges have length one). Let $n$ be a positive integer, and let $d_{\ell \infty}$ be the $\ell^{\infty}$-distance on $Y^{n}: d_{\ell \infty}(x, y):=\max _{i} d\left(x_{i}, y_{i}\right)$. It is known that $Y^{n}$ cannot be topologically embedded into $\mathbb{R}^{2 n-1}$. A proof of this result based on the Borsuk-Ulam theorem can be found in A.B. Skopenkov [10, pp. 287-288], and more general results on the problem of embedding products of graphs into the Euclidean spaces in M. Skopenkov [11]. The purpose of this subsection is to prove an $\varepsilon$-embedding version of the above result: 
Proposition 2.5. For any $0<\varepsilon<1$, there does not exist an $\varepsilon$-embedding from $\left(Y^{n}, d_{\ell \infty}\right)$ to $\mathbb{R}^{2 n-1}$.

It is likely this proposition is known to some specialists. The proof below is an application of the method in Matoušek's book [9, Chapter 5]; probably it also follows from the method of [10].

The most important ingredient of the proof is the following form of the Borsuk-Ulam theorem $\left[9\right.$, p. 23, (BU2a)]: There does not exist a $\mathbb{Z}_{2}$-equivariant continuous map from $S^{n}$ to $S^{n-1}$. Here $\mathbb{Z}_{2}=\mathbb{Z} / 2 \mathbb{Z}$, and it acts on $S^{n}$ by the antipodal map.

Let $K$ be a (geometric) simplicial complex; in this subsection we consider only finite simplicial complexes. For a point $x \in K$ we denote by $\operatorname{supp}(x)$ the simplex of $K$ containing $x$ in its relative interior. Let $K^{* 2}:=K * K$ be the join of the two copies of $K$, and define the deleted join $K_{\Delta}^{* 2} \subset K^{* 2}$ by

$$
K_{\Delta}^{* 2}:=\left\{(1-t) x_{1} \oplus t x_{2} \mid 0 \leq t \leq 1, x_{1}, x_{2} \in K, \operatorname{supp}\left(x_{1}\right) \cap \operatorname{supp}\left(x_{2}\right)=\emptyset\right\} .
$$

By convention, for any $x \in K$ both $x \oplus 0$ and $0 \oplus x$ are contained in $K_{\Delta}^{* 2}$. The group $\mathbb{Z}_{2}$ freely acts on this space by $(1-t) x_{1} \oplus t x_{2} \mapsto t x_{2} \oplus(1-t) x_{1}$. For example, $\left(D_{3}\right)_{\Delta}^{* 2}$ is $\mathbb{Z}_{2}$-homeomorphic to $S^{1}$.

The following fact is easy to prove (see [9, 5.5.2 Lemma]): Let $K$ and $L$ be simplicial complexes. Then we have a $\mathbb{Z}_{2}$-homeomorphism:

$$
(K * L)_{\Delta}^{* 2} \cong K_{\Delta}^{* 2} * L_{\Delta}^{* 2} .
$$

(In the right-hand-side, $\mathbb{Z}_{2}$ acts on $K_{\Delta}^{* 2}$ and $L_{\Delta}^{* 2}$ simultaneously.) The following lemma is proved in $[9,5.5 .4$ Lemma].

Lemma 2.6. Set

$$
R_{n}:=\left(\mathbb{R}^{n}\right)^{* 2} \backslash\left\{\frac{1}{2} x \oplus \frac{1}{2} x \mid x \in \mathbb{R}^{n}\right\} .
$$

$\mathbb{Z}_{2}$ acts on $R_{n}$ as in the above. Then there is a $\mathbb{Z}_{2}$-equivariant continuous map from $R_{n}$ to $S^{n}$.

Following Skopenkov [11, p. 193], we shall make use of the following fact:

Lemma 2.7. Let $K$ and $L$ be simplicial complexes, and $C K$ and $C L$ be their cones. Then there is a homeomorphism $f: C K \times C L \rightarrow C(K * L)$ such that for any simplex $\Delta \subset$ $C(K * L)$ its preimage $f^{-1}(\Delta)$ is a union of (at most two) sets of the form $\Delta_{1} \times \Delta_{2}\left(\Delta_{1}\right.$, $\Delta_{2}$ are simplexes of $C K, C L$ respectively). Here we use the natural simplicial complex structures of $C K, C L$ and $C(K * L)$.

Proof. Take a homeomorphism

$$
\varphi:[0,1] \times[0,1] \rightarrow\{(S, T) \mid S \geq 0, T \geq 0, S+T \leq 1\}, \quad(s, t) \mapsto(S, T),
$$


such that $\varphi(\{s=0\})=\{S=0\}, \varphi(\{t=0\})=\{T=0\}$ and $\varphi(\{s=1\} \cup\{t=1\})=$ $\{S+T=1\}$. Let $p, p^{\prime}, p^{\prime \prime}$ be the base point of the cones $C(K * L), C(K)$, and $C(L)$, so that we may identify $C(K * L)$ with $\{p\} * K * L$ (and similarly for $p^{\prime}, p^{\prime \prime}$ and $C(K), C(L)$ ). We define $f: C K \times C L \rightarrow C(K * L)=\{p\} * K * L$ by

$$
f([s x],[t y]):=(1-S-T) p \oplus S x \oplus T y, \quad(0 \leq s, t \leq 1, \varphi(s, t)=(S, T), x \in K, y \in L) .
$$

From $\varphi(\{s=0\})=\{S=0\}$ and $\varphi(\{t=0\})=\{T=0\}$, the map $f$ is well-defined. Since $\varphi$ is a homeomorphism, so is $f$. If $\Delta$ is a simplex in $C(K)$ (which we identify with the appropriate simplex in $C(K * L))$, then $f^{-1}(\Delta)=\Delta \times\left\{p^{\prime \prime}\right\}$ and similarly for $\Delta \subset C(L)$, $f^{-1}(\Delta)=\left\{p^{\prime}\right\} \times \Delta$. Otherwise, $\Delta \subset C(K * L)$ may either contain $p$ in which case it has the form $C\left(\Delta_{1} * \Delta_{2}\right)$ or it does not in which case it has the form $\Delta_{1} * \Delta_{2}$ with $\Delta_{1} \subset K$ and $\Delta_{2} \subset L$. In the first case, $f^{-1}\left(C\left(\Delta_{1} * \Delta_{2}\right)\right)=C\left(\Delta_{1}\right) \times C\left(\Delta_{2}\right)$ whereas in the second

$$
f^{-1}\left(\Delta_{1} * \Delta_{2}\right)=C\left(\Delta_{1}\right) \times \Delta_{2} \cup \Delta_{1} \times C\left(\Delta_{2}\right)
$$

Proof of Proposition 2.5. $Y=C\left(D_{3}\right)$. By iterated applications of Lemma 2.7, there is a homeomorphism $f: Y^{n} \rightarrow C\left(\left(D_{3}\right)^{* n}\right)=: K$ such that for any simplex $\Delta \subset K$ its preimage $f^{-1}(\Delta)$ is a union of sets of the form $\Delta_{1} \times \cdots \times \Delta_{n}\left(\Delta_{1}, \ldots, \Delta_{n}\right.$ are simplexes of $\left.Y\right)$. This property implies: if $x, y \in K$ satisfy $\operatorname{supp}(x) \cap \operatorname{supp}(y)=\emptyset$ then $d_{\ell \infty}\left(f^{-1}(x), f^{-1}(y)\right) \geq 1$. (Note that the distance between two disjoint simplexes of $Y$ is greater than or equal to 1.)

Suppose that there is an $\varepsilon$-embedding $\varphi:\left(Y^{n}, d_{\ell^{\infty}}\right) \rightarrow \mathbb{R}^{2 n-1}$ for $0<\varepsilon<1$. Then for any two points $x, y \in K$ with $\operatorname{supp}(x) \cap \operatorname{supp}(y)=\emptyset$ we have $\varphi \circ f^{-1}(x) \neq \varphi \circ f^{-1}(y)$. Then we can define a $\mathbb{Z}_{2}$-equivariant continuous map from the deleted join $K_{\Delta}^{* 2}$ to $R_{2 n-1}$ (defined in Lemma 2.6) by $(1-t) x \oplus t y \mapsto(1-t) \varphi \circ f^{-1}(x) \oplus t \varphi \circ f^{-1}(y)$. Hence by Lemma 2.6 there is a $\mathbb{Z}_{2}$-equivariant continuous map from $K_{\Delta}^{* 2}$ to $S^{2 n-1}$. On the other hand, we have the following $\mathbb{Z}_{2}$-homeomorphisms:

$$
K_{\Delta}^{* 2}=\left(\{p\} *\left(D_{3}\right)^{* n}\right)_{\Delta}^{* 2} \cong(\{p\})_{\Delta}^{* 2} *\left\{\left(D_{3}\right)_{\Delta}^{* 2}\right\}^{* n} \cong S^{0} *\left(S^{1}\right)^{* n} \cong S^{2 n}
$$

Here we have used the identification of $K=C\left(\left(D_{3}\right)^{* n}\right)$ with the join of $\left(D_{3}\right)^{* n}$ with a one-point space $\{p\}$, the identity (1), as well as the $\mathbb{Z}_{2}$-homeomorphisms $(\{p\})_{\Delta}^{* 2} \cong S^{0}$, $\left(D_{3}\right)_{\Delta}^{* 2} \cong S^{1}$ and $S^{l} * S^{m} \cong S^{l+m+1}$. Therefore we conclude that there is a $\mathbb{Z}_{2}$-equivariant continuous map from $S^{2 n}$ to $S^{2 n-1}$. But this contradicts the Borsuk-Ulam theorem.

\section{Proof of the MAIN THEOREM}

The construction of $X$ below is based on Lindenstrauss-Weiss [8, pp. 10-11]. Let $Y$ be the triod graph. Let $D$ be a positive integer, and set $K:=Y^{D}$. Let $d$ be the graph distance on $Y$, and let $d_{\ell \infty}$ be the $\ell^{\infty}$-distance on $K=Y^{D}$ introduced in Section 2.2. We 
define a distance on $K^{\mathbb{Z}}$ by

$$
\operatorname{dist}(x, y):=\sum_{n \in \mathbb{Z}} 2^{-|n|} d_{\ell \infty}\left(x_{n}, y_{n}\right) .
$$

Let $\sigma: K^{\mathbb{Z}} \rightarrow K^{\mathbb{Z}}$ be the shift transformation.

Fix a sequence of rational numbers $p_{n}(n \geq 1)$ such that

$$
\prod_{n=1}^{\infty}\left(1-p_{n}\right)=\frac{1}{2}, \quad 0<p_{n}<1 .
$$

We will construct the following three objects satisfying the conditions (i)-(iv) below:

- A decreasing sequence of closed shift-invariant subsets of $K^{\mathbb{Z}}$ :

$$
K^{\mathbb{Z}}=X_{0} \supset X_{1} \supset X_{2} \supset X_{3} \supset \ldots, \quad X:=\bigcap_{n=0}^{\infty} X_{n} .
$$

- A sequence of integers:

$$
0=a_{0}<b_{0}<a_{1}<b_{1}<\cdots<a_{n}<b_{n}<a_{n+1}<b_{n+1}<\ldots
$$

- Closed subsets $B_{n} \subset K^{b_{n}}(n \geq 1)$.

(i) $a_{0}=0$ and $b_{0}=1 . p_{n}=a_{n} / b_{n}(n \geq 1) \cdot b_{n} \mid b_{n+1}$ and $b_{n} \mid a_{n+1}$. Moreover

$$
b_{n}\left(2 \prod_{k \leq n}\left(1-p_{k}\right)-1\right) \rightarrow \infty \quad \text { as } n \rightarrow \infty .
$$

(ii) $X_{n}$ is the block-type space defined by $B_{n}$ :

$$
X_{n}=\left\{x \in K^{\mathbb{Z}} \mid \exists k \in \mathbb{Z} \forall l \in \mathbb{Z}: x_{k+l b_{n}}^{k+l b_{n}+b_{n}-1} \in B_{n}\right\} .
$$

$B_{0}=K$ and $X_{0}=K^{\mathbb{Z}}$.

(iii) We define a decreasing sequence $\mathbb{Z}=I_{0} \supset I_{1} \supset I_{2} \supset \ldots$ by

$$
I_{n}:=\left\{x \in \mathbb{Z} \mid \forall 0 \leq k \leq n, \exists j \in\left\{0,1, \ldots, b_{k}-a_{k}-1\right\}: x \equiv j \bmod b_{k}\right\} .
$$

We identify $K^{b_{n}}$ with $K^{\left\{0,1,2, \ldots, b_{n}-1\right\}}$, and let $\pi_{n}: K^{b_{n}} \rightarrow K^{\left\{0,1,2, \ldots, b_{n}-1\right\} \backslash I_{n}}$ be the projection. Then there is $x(n) \in K^{\left\{0,1,2, \ldots, b_{n}-1\right\} \backslash I_{n}}$ such that $B_{n}=\pi_{n}^{-1}(x(n))(n \geq 1)$. The sequence $\{x(n)\}_{n \geq 1}$ satisfies the following compatibility condition: If $k \in\left\{0,1,2, \ldots, b_{n+1}-1\right\} \backslash I_{n+1}$ and $k^{\prime} \in\left\{0,1,2, \ldots, b_{n}-1\right\} \backslash I_{n}$ satisfy $k \equiv k^{\prime} \bmod b_{n}$, then $x(n+1)_{k}=x(n)_{k^{\prime}}$. This condition is equivalent to $B_{n+1} \subset \underbrace{B_{n} \times B_{n} \times \cdots \times B_{n}}_{b_{n+1} / b_{n}}$ (and hence $X_{n+1} \subset X_{n}$ ).

(iv) For any $x, y \in X_{n}(n \geq 1)$ there is $k \in \mathbb{Z}$ satisfying $\operatorname{dist}\left(\sigma^{k}(x), y\right) \leq 2^{-n}$.

From the condition (iv) it easily follows that the system $(X, \sigma)$ is minimal. Set $I:=$ $\bigcap_{n=0}^{\infty} I_{n} \subset \mathbb{Z}$. From the condition (i), for $n<m, b_{n} \mid a_{m}, b_{m}$ and hence $b_{n} \leq b_{m}-a_{m}$. So $I \cap\left\{0,1,2, \ldots, b_{n}-1\right\}=I_{n} \cap\left\{0,1,2, \ldots, b_{n}-1\right\}$. For each $n \geq 1$,

$$
\left|I_{n+1} \cap\left\{0,1,2, \ldots, b_{n+1}-1\right\}\right|=\frac{b_{n+1}-a_{n+1}}{b_{n}}\left|I_{n} \cap\left\{0,1,2, \ldots, b_{n}-1\right\}\right| .
$$


Hence

$$
\left|I \cap\left\{0,1,2, \ldots, b_{n}-1\right\}\right|=\left|I_{n} \cap\left\{0,1,2, \ldots, b_{n}-1\right\}\right|=b_{n} \prod_{k=1}^{n}\left(1-p_{k}\right) .
$$

Lemma 3.1. Under the above conditions, for any $n \geq 1$, there is a continuous distanceincreasing map from $\left(Y^{D b_{n} \prod_{k=1}^{n}\left(1-p_{k}\right)}, d_{\ell \infty}\right)$ to $\left(X\right.$, dist $\left._{b_{n}}\right)$.

Proof. We have $Y^{D b_{n} \prod_{k=1}^{n}\left(1-p_{k}\right)}=K^{I \cap\left\{0,1,2, \ldots, b_{n}-1\right\}}$. Fix a point $p \in K$. We define a map $K^{I \cap\left\{0,1,2, \ldots, b_{n}-1\right\}} \rightarrow X$ by mapping each $x \in K^{I \cap\left\{0,1,2, \ldots, b_{n}-1\right\}}$ to the point $x^{\prime} \in X$ defined by

$$
x_{k}^{\prime}:= \begin{cases}x_{k} & \left(k \in I \cap\left\{0,1,2, \ldots, b_{n}-1\right\}\right) \\ x(m)_{k^{\prime}} & \left(\exists m \geq 1, \exists k^{\prime} \in\left\{0,1,2, \ldots, b_{m}-1\right\} \backslash I_{m}: k^{\prime} \equiv k \bmod b_{m}\right) \\ p & \text { (otherwise) }\end{cases}
$$

From the compatibility condition on $\{x(m)\}$, this map is well-defined. We can easily check that this is continuous and distance-increasing.

Lemma 3.2. $\operatorname{mdim}(X, \sigma)=D / 2$.

Proof. We have $\operatorname{dim} B_{n}=D\left|I_{n} \cap\left\{0,1,2, \ldots, b_{n}-1\right\}\right|=D b_{n} \prod_{k=1}^{n}\left(1-p_{k}\right)$. From Lemma 2.4 ,

$$
\operatorname{mdim}(X, \sigma) \leq \operatorname{mdim}\left(X_{n}, \sigma\right) \leq D \prod_{k=1}^{n}\left(1-p_{k}\right) .
$$

Letting $n \rightarrow \infty$ and using (2), we get the upper bound $\operatorname{mdim}(X, \sigma) \leq D / 2$. On the other hand, from Lemma 3.1, for $0<\varepsilon<1$,

$$
\frac{\mathrm{Widim}_{\varepsilon}\left(X, \operatorname{dist}_{b_{n}}\right)}{b_{n}} \geq \frac{\mathrm{Widim}_{\varepsilon}\left(Y^{D b_{n} \prod_{k=1}^{n}\left(1-p_{k}\right)}, d_{\ell}\right)}{b_{n}}=D \prod_{k=1}^{n}\left(1-p_{k}\right) .
$$

Here we have used Example 2.1 and the fact that the space $\left(Y^{D b_{n} \prod_{k=1}^{n}\left(1-p_{k}\right)}, d_{\ell^{\infty}}\right)$ contains $\left([0,1]^{D b_{n} \prod_{k=1}^{n}\left(1-p_{k}\right)}, d_{\ell}\right)$. Letting $n \rightarrow \infty$ and $\varepsilon \rightarrow 0$, we get $\operatorname{mdim}(X, \sigma) \geq D / 2$.

Lemma 3.3. $(X, \sigma)$ cannot be embedded into $\left(\left([0,1]^{D}\right)^{\mathbb{Z}}, \sigma\right)$.

Proof. Suppose that there is an embedding $f$ from $(X, \sigma)$ into $\left(\left([0,1]^{D}\right)^{\mathbb{Z}}, \sigma\right)$. Take a distance $d^{\prime}$ on $\left([0,1]^{D}\right)^{\mathbb{Z}}$. There exists $\varepsilon>0$ such that if $d^{\prime}(f(x), f(y)) \leq \varepsilon$ then $\operatorname{dist}(x, y) \leq$ $1 / 2$. Since $f$ commutes with the shift transformations, for every $N \geq 1$, if two points $x, y \in X$ satisfy $d_{N}^{\prime}(f(x), f(y)) \leq \varepsilon$ then $\operatorname{dist}_{N}(x, y) \leq 1 / 2$. We can take a positive integer $L=L(\varepsilon)$ such that if two points $x, y \in\left([0,1]^{D}\right)^{\mathbb{Z}}$ satisfy $x_{n}=y_{n}$ for $-L \leq n \leq L$ then $d^{\prime}(x, y) \leq \varepsilon$. Then, for every $N \geq 1$, if two points $x, y \in\left([0,1]^{D}\right)^{\mathbb{Z}}$ satisfy $x_{n}=y_{n}$ for $-L \leq n \leq N+L$ then $d_{N}^{\prime}(x, y) \leq \varepsilon$.

Let $\pi_{[-L, N+L]}:\left([0,1]^{D}\right)^{\mathbb{Z}} \rightarrow\left([0,1]^{D}\right)^{\{-L,-L+1, \ldots, N+L\}}$ be the natural projection. Then the map $\pi_{[-L, N+L]} \circ f:\left(X, \operatorname{dist}_{N}\right) \rightarrow\left([0,1]^{D}\right)^{\{-L,-L+1, \ldots, N+L\}}$ becomes a $1 / 2$-embedding. 
Using Lemma 3.1, we conclude that for any $n \geq 1$ there exists a $1 / 2$-embedding from $\left(Y^{D b_{n} \prod_{k=1}^{n}\left(1-p_{k}\right)}, d_{\ell^{\infty}}\right)$ to $[0,1]^{D\left(b_{n}+2 L+1\right)}$. Then Proposition 2.5 implies

$$
D\left(b_{n}+2 L+1\right) \geq 2 D b_{n} \prod_{k=1}^{n}\left(1-p_{k}\right)
$$

hence $b_{n}\left(2 \prod_{k=1}^{n}\left(1-p_{k}\right)-1\right) \leq 2 L+1$ which for $n$ large contradicts $(3)$.

The last problem is to define $X_{n}, a_{n}, b_{n}$ and $B_{n}$. (Recall that we fixed rational numbers $p_{n}$ satisfying (2).) We construct them by induction. First we set $X_{0}:=K^{\mathbb{Z}}, a_{0}:=0$, $b_{0}:=1, B_{0}:=K$. Suppose that we have constructed $X_{n}, a_{n}, b_{n}, B_{n}$. Since block-type systems are topologically transitive, there is $\tilde{x} \in X_{n}$ whose orbit is dense in $X_{n}$. We can assume that $\tilde{x}_{l b_{n}}^{l b_{n}+b_{n}-1} \in B_{n}$ for all integers $l$.

Take a positive integer $L$ such that for any $x \in X_{n}$ there is $k \in[-L, L]$ satisfying $\operatorname{dist}\left(\sigma^{k}(\tilde{x}), x\right) \leq 2^{-n-2}$. We take a positive even integer $a_{n+1}>b_{n}$ sufficiently large so that

- $b_{n} \mid\left(a_{n+1} / 2\right)$.

- $a_{n+1} \gg L$. $\left(a_{n+1} \geq 2 L+2 n+10\right.$ will do. But the precise estimate is not important.)

- There is a positive integer $b_{n+1}$ such that $b_{n}<b_{n+1}, b_{n} \mid b_{n+1}, p_{n+1}=a_{n+1} / b_{n+1}$, and

$$
b_{n+1}\left(2 \prod_{k \leq n+1}\left(1-p_{k}\right)-1\right) \geq n+1 .
$$

Then $a_{n+1}$ and $b_{n+1}$ satisfy the condition (i).

We define $B_{n+1} \subset K^{b_{n+1}}$ as the set of $x \in K^{b_{n+1}}$ satisfying

$$
x_{l b_{n}}^{l b_{n}+b_{n}-1} \in B_{n}\left(\forall l \in \mathbb{Z} \text { with } 0 \leq l<b_{n+1} / b_{n}\right), \quad x_{b_{n+1}-a_{n+1}}^{b_{n+1}-1}=\tilde{x}_{-a_{n+1} / 2}^{a_{n+1} / 2-1} .
$$

Here $x_{m}^{n}=\left(x_{m}, x_{m+1}, \ldots, x_{n}\right)$ for $m \leq n$ and $x=\left(x_{0}, x_{1}, \ldots, x_{b_{n+1}-1}\right) \in K^{b_{n+1}}$. Let $X_{n+1}$ be the block-type system defined by $B_{n+1}$ (condition (ii)). From the definition of $\tilde{x}$ and $a_{n+1} \gg L$, the system $X_{n+1}$ satisfies the condition (iv). We define $x(n+1) \in$ $K^{\left\{0,1,2, \ldots, b_{n+1}-1\right\} \backslash I_{n+1}}$ (see the condition (iii)) by

$$
x(n+1)_{k}:= \begin{cases}x(n)_{k^{\prime}} & \left(\exists k^{\prime} \in\left\{0,1,2, \ldots, b_{n}-1\right\} \backslash I_{n}: k \equiv k^{\prime} \bmod b_{n}\right) \\ \tilde{x}_{k-b_{n+1}+a_{n+1} / 2} & \left(b_{n+1}-a_{n+1} \leq k \leq b_{n+1}-1\right) .\end{cases}
$$

Since we assume $\tilde{x}_{l b_{n}}^{l b_{n}+b_{n}-1} \in B_{n}$ for all integers $l$, this is well-defined. (When $n=0$, we set $x(1)_{k}=\tilde{x}_{k-b_{1}+a_{1} / 2}$ for $b_{1}-a_{1} \leq k \leq b_{1}-1$.) We can easily check that the condition (iii) is satisfied. This completes the proof of Theorem 1.3.

\section{REFERENCES}

[1] J. Auslander, Minimal flows and their extensions, North-Holland, Amsterdam (1988)

[2] M. Coornaert, F. Krieger, Mean topological dimension for actions of discrete amenable groups, Discrete Contin. Dyn. Syst. 13 (2005) 779-793 
[3] M. Gromov, Topological invariants of dynamical systems and spaces of holomorphic maps: I, Math. Phys. Anal. Geom. 2 (1999) 323-415

[4] Y. Gutman, Embedding $\mathbb{Z}^{k}$-actions in cubical shifts and $\mathbb{Z}^{k}$-symbolic extensions, Ergodic Theory Dynam. Systems 31 (2011) 383-403

[5] W. Hurewicz, H. Wallman, Dimension theory, Princeton University Press, Princeton (1941)

[6] A. Jaworski, Ph.D. Thesis, University of Maryland (1974)

[7] E. Lindenstrauss, Mean dimension, small entropy factors and an embedding theorem, Inst. Hautes Études Sci. Publ. Math. 89 (1999) 227-262

[8] E. Lindenstrauss, B. Weiss, Mean topological dimension, Israel J. Math. 115 (2000) 1-24

[9] J. Matoušek, Using the Borsuk-Ulam theorem, Lectures on topological methods in combinatorics and geometry, Corrected 2nd printing, Springer-Verlag, Berlin Heidelberg (2008)

[10] A. B. Skopenkov, Embedding and knotting of manifolds in Euclidean spaces, Surveys in contemporary mathematics, Cambridge University Press, Cambridge (2008) 248-342

[11] M. Skopenkov, Embedding products of graphs into Euclidean spaces, Fund. Math. 179 (2003) 191-198

Elon Lindenstrauss

Einstein Institute of Mathematics, Hebrew University, Jerusalem 91904, Israel

E-mail address: elon@math.huji.ac.il

Masaki Tsukamoto

Department of Mathematics, Kyoto University, Kyoto 606-8502, Japan

E-mail address: tukamoto@math.kyoto-u.ac.jp 